\title{
ОФЭКТ/КТ В АЛГОРИТМЕ ПРЕДОПЕРАЦИОННОЙ ДИАГНОСТИКИ ПЕРВИЧНОГО ГИПЕРПАРАТИРЕОЗА
}

\author{
Слащук К.Ю., Дегтярев М.В., Серженко С.С. \\ ФГБУ «НМИЦ эндокринологии» Минздрава России, Москва
}

\begin{abstract}
АКТУАЛЬНОСТЬ: гиперпаратиреоз (ГПТ) занимает третье место в структуре патологий эндокринной системы после заболеваний щитовидной железы и сахарного диабета. Подавляющее большинство случаев приходится на первичный гиперпаратиреоз (ПГПТ), который более чем в 85-90\% является спорадическим. Заболеваемость может достигать 1-2\% населения в старшей возрастной группе (>55 лет). Ранняя диагностика и лечение ПГПТ позволяют избежать тяжелых поражений скелета, почек, других органов и систем, тем самым улучшая качество жизни пациентов и снижая частоту инвалидизации пациентов. Единственным радикальным методом лечения ПГПТ является хирургическое удаление патологически измененных околощитовидных желез (ОЩЖ).

ЦЕЛЬ: оценить диагностическую точность метода ОФЭКТ/КТ в алгоритме топической визуализации ОЩЖ при планировании минимально инвазивной паратиреоидэктомии.

МАТЕРИАЛЫ И МЕТОДЫ: в перИод с 2017 по 2020 годы, было обследован 200 пациентов с входящим диагнозом ПГПТ, в возрасте от 21 до 82 лет (средний возраст 57 лет). После первичного обследования, 189 пациентов с лабораторно верифицированным ПГПТ, лабораторные данные (медиана параметров): ПТГ 140,9 пг/мл; кальций общ. 2,8 ммоль/л; кальций ион. 1,38 ммоль/л; 25OH-Витамин D 15,38 нг/мл, прошли предоперационную топическую диагностику, были выполнены сцинтиграфия в режиме ОФЭКТ/KT (GE Discovery NM/CT 670), при необходимости с в/в контрастированием, в сочетании с УзИ щитовидной и околощитовидных желез (GE Voluson E8). C последующим хирургическим лечением в объеме минимально инвазивной парааденомэктомии во всех случаях, когда это было возможно, с последующим морфологическим исследованием.
\end{abstract}

РЕЗУЛЬТАТЫ И ВЫВОДЫ: уровни ПТГ и кальция крови нормализовались у 186 из 189 оперированных пациентов (98\%). При анализе операционных характеристик методов визуализации: общая точность УЗИ шеи, планарной сцинтиграфии и ОФЭКТ/КТ при визуализации ОЩЖ составила 89\%, 82\% и 98\% соответственно; специфичность 54\%, 92\%, 85\%; чувствительность - 91\%, 81\% и 99\% соответственно; прогностическая ценность положительного результата методов была сопоставима для всех методов 97-99\%; прогностическая ценность отрицательная результата составила 29\%, 25\% и 85\% соответственно.

Таким образом, ОФЭКТ/КТ в сочетании сУзИ шеи, по данным нашего исследования, является наиболее точной комбинацией методов предоперационной топической диагностики образований ОЩЖ при ПГПт. При совпадении результатов двух исследований нет необходимости в выполнении каких-либо дополнительных диагностических тестов. В случае отрицательного или дискордантного диагностического результата рекомендуются дополнительные исследования (одномоментное с ОФЭКТ/КТ в/в контрастирование либо последовательное МСКТ с в/в контрастированием вторым этапом, ТАБ со смывом из иглы на ПТГ при наличии объекта для биопсии. Основные факторы, затрудняющие диагностику, - заболевания щитовидной железы, в первую очередь функциональные (диффузный и узловой токсический зоб, узловой и многоузловой зоб на фоне ХАИТ, рак щитовидной железы и его метастазы в л/у шеи, фолликулярная аденома щитовидной железы), малые размеры ОЩЖ (объем $\leq 0,42 \mathrm{~cm}^{3}$ ) и интратиреоидное расположение ОЩЖ. 